\title{
Malófagos (Phthiraptera, Amblycera, Ischnocera) em aves cativas no sudeste do Brasil
}

\author{
Salete Oliveira da Silva ${ }^{1}$, Heloiza Helena de Oliveira $^{1}$, Rodrigo Hidalgo Friciello Teixeira² \& Marinete Amorim ${ }^{1}$
}

1Laboratório de Ixodides-Referência Nacional em Vetores das Riquétsias, Instituto Oswaldo Cruz, Fundação Oswaldo Cruz, $21045-900$ Rio de
Janeiro-RJ, Brasil. saleteoliveiradasilva@ gmail.com; hel.hel@ terra.com.br
${ }^{2}$ Parque Zoológico Municipal Quinzinho de Barros, 18021-020 Sorocaba-SP, Brasil.

\begin{abstract}
Chewing lice (Phthiraptera, Amblycera, Ischnocera) on captive birds in southeastern Brazil. Twelve chewing lice species were identified in Parque Zoológico Municipal Quinzinho de Barros, Sorocaba and Fundação Jardim Zoológico, Rio de Janeiro. The parasites found were: Ciconiphilus pectiniventris in Cygnus atratus (Anseriformes, Anatidae); Kurodaia sp. in Buteo albicaudatus (Falconiformes, Accipitridae); Degeeriella sp. in Falco sparverius (Falconiformes, Falconidae); Colpocephalum sp. and Goniocotes parviceps in Pavo cristatus (Galliformes, Phasianidae); Goniodes pavonis in Rhea americana (Rheiformes, Rheidae); Colpocephalum cristatae and Heptapsogaster sp. in Cariama cristata (Gruiformes, Cariamidae); Austrophilopterus cancellosus in Ramphastos dicolorus (Piciformes, Ramphastidae); Strigiphilus crucigerus in Otus choliba (Strigiformes, Strigidae); Kurodaia sp. in Rhinoptynx clamator (Strigiformes, Strigidae) and Colpocephalum pectinatum in Speotyto cunicularia (Strigiformes, Strigidae). The host-lice relationships are new in Strigiformes in Brazil.
\end{abstract}

KEYWORDS. Chewing lice; Colpocephalum cristatae; host-lice relationship.

RESUMO. Malófagos (Phthiraptera, Amblycera, Ischnocera) em aves cativas no sudeste do Brasil. Foram identificadas 12 espécies de malófagos no Parque Zoológico Municipal Quinzinho de Barros, Sorocaba e Fundação Jardim Zoológico, Rio de Janeiro. Ciconiphilus pectiniventris em Cygnus atratus (Anseriformes, Anatidae); Kurodaia sp. em Buteo albicaudatus (Falconiformes, Accipitridae); Degeeriella sp. em Falco sparverius (Falconiformes, Falconidae); Colpocephalum sp. e Goniocotes parviceps em Pavo cristatus (Galliformes, Phasianidae); Goniodes pavonis em Rhea americana (Rheiformes, Rheidae); Colpocephalum cristatae e Heptapsogaster sp. em Cariama cristata (Gruiformes, Cariamidae); Austrophilopterus cancellosus em Ramphastos dicolorus (Piciformes, Ramphastidae); Strigiphilus crucigerus em Otus choliba (Strigiformes, Strigidae); Kurodaia sp. em Rhinoptynx clamator (Strigiformes, Strigidae) e Colpocephalum pectinatum em Speotyto cunicularia (Strigiformes, Strigidae). As relações parasito hospedeiros em Strigiformes são novas no Brasil.

PALAVRAS-CHAVE. Colpocephalum cristatae; piolhos mastigadores; parasito-hospedeiro.

As aves possuem um grande número de artrópodes parasitas, como ácaros plumícolas (Acari: Acaridida), carrapatos (Acari: Ixodida) e malófagos (Insecta: Phthiraptera).

Os malófagos são insetos ápteros, ectoparasitos permanentes, de corpos achatados dorso-ventralmente, podem medir de $0.8 \mathrm{~mm}$ a $11 \mathrm{~mm}$, são hemimetábolos e todos os instares vivem sobre os hospedeiros (Johnson \& Clayton 2003).

Amostragens rotineiras de malófagos em aves apreendidas ou cativas poderão fornecer melhor entendimento sobre a diversidade da fauna dos Phthiraptera no Brasil, dado a dificuldade de captura de material na natureza. Por outro lado, a coleta e estudo de material proveniente de aves de vida livre poderiam elucidar aspectos da ecologia e comportamento destes parasitos (Valim et al. 2005). No Brasil poucos trabalhos tem dado ênfase a esses parasitos de aves cativas (Freitas et al. 2002; Silva et al. 2004; Valim et al. 2005).

Este trabalho tem como objetivo estudar amostras de malófagos coletadas em aves confinadas em cativeiros da Fundação Jardim Zoológico (RioZoo), Rio de Janeiro, Brasil e do Parque Zoológico Municipal Quinzinho de Barros (PZMQB), Sorocaba, Brasil.

Os piolhos foram montados em preparações permanentes e encontram-se depositados na Coleção de Artrópodes Vetores Ápteros de Importância em Saúde das Comunidades, inserida na Coleção Entomológica da Fundação Oswaldo Cruz. A nomenclatura utilizada para identificação dos malófagos e associações da relação parasita/hospedeiro estão de acordo com Price et al. (2003). Os nomes científicos e vulgares seguem Sick (1997).

Foi identificado um total de 64 espécimes de malófagos, sendo 14 machos e 50 fêmeas. Os dados foram agrupados por subordem, família e espécies dos Phthiraptera, com os dados de captura: 


\section{Amblycera \\ Menoponidae \\ Ciconiphilus pectiniventris (Harrison, 1916)}

Material examinado: 1 macho e 6 fêmeas, em Cygnus atratus (Latham, 1790) (Anseriformes, Anatidae), 20.I.2000, Teixeira. R.H.F., col. PZMQB.

Nota. Foi identificado através da chave dicotômica de Price \& Beer (1965), sendo este um novo relato para literatura brasileira. Price \& Graham (1997) relataram os mesmos achados dos autores acima citados, tendo observado várias espécies de aves das Ordens Ciconiformes e Anseriformes hospedeiros desta espécie, sendo condizente com a lista mundial de Price et al. (2003). Esta espécie de ave é exótica à fauna brasileira (Sick 1997). Por isso, podemos considerar que C. pectiniventris é uma espécie introduzida no Brasil.

\section{Colpocephalum cristatae Price, 1968}

Material examinado: 1 macho e 7 fêmeas, em Cariama cristata (Linnaeus, 1766) (Gruiformes, Cariamidae), 09.XI.2001, Teixeira, R.H.F., Col. PZMQB.

Nota. Trata-se de um novo relato para literatura brasileira, muito embora Brum et al. (2003) já haviam identificado Colpocephalum parasitando Cariama cristata no Rio Grande do Sul, mas apenas a nível genérico. Segundo Price et al. (2003) esta espécie pode parasitar C. cristata e Chunga burmeisteri (Hartlaub, 1860).

\section{Colpocephalum pectinatum Osborn, 1902}

Material examinado: 1 macho e 1 fêmea, em Speotyto cunicularia (Molina, 1782) (Strigiformes, Strigidae), 10.VIII.1995, Teixeira, R.H.F., col. PZMQB.

Nota. Esta informação está de acordo com Price \& Beer (1963) que indicam essa relação parasito-hospedeiro, entretanto, ainda não havia sido reportado para o Brasil. Price et al. (2003) relatam as seguintes aves parasitadas por esta espécie: Athene brama (Termminck,1821), Athene cunicularia (Molina, 1782), Athene cunicularia hypugaeae (Bonaparte,1825), Otus bakkamoera Pennant, 1769, Otus watsoni (Cassin, 1849) e Tyto alba (Scopoli, 1769).

\section{Colpocephalum sp.}

Material examinado: 1 macho e 1 fêmea, Pavo cristatus Linnaeus, 1758 (Galliformes, Phasianidae), 17.III.2000, Teixeira, R.H.F., col. PZMQB.

Nota. Price et al. (2003) relataram as seguintes espécies de Colpocephalum parasitando Pavo cristatus: Colpocephalum tausi (Ansari, 1951) e Colpocephalum toracicum Kellogg \& Paine, 1914.

\section{Kurodaia sp.}

Material examinado: 5 fêmeas, Rhinoptynx clamator (Vieillot, 1816) (Strigiformes, Strigidae), 06.II.1994, Teixeira, R.H.F., col. PZMQB.
Nota. Esses dados são concordantes com Clayton (1990) e Price et al. (2003) que registraram a presença de Kurodaia em corujas. No Brasil foi relatado pela primeira vez Strigiphilus heterurus Carriker, 1966 em Rhinoptynx clamator por Valim et al. (2005).

\section{Kurodaia sp.}

Material examinado: 4 machos e 13 fêmeas, Buteo albicaudatus (Vieillot, 1816) (Falconiformes, Accipitridae), 23.III.1994, Fedulo, L. P., col. RioZoo.

Nota. Segundo Dickinson (2003) esta espécie pode também ser parasitada por Colpocephalum turbinatum Denny, $1842 \mathrm{e}$ Degeeriella regalis (Giebel, 1866).

\section{Ischnocera \\ Philopteridae \\ Austrophilopterus cancellosus (Carriker, 1903)}

Material examinado: 1 macho e 9 fêmeas, em Ramphastos dicolorus (Linnaeus, 1766) (Piciformes, Ramphastidae), 27.XII.2001, Teixeira, R.H.F., col. PZMQB.

Nota. Price et al. (2003) relataram Austrophilopterus parasitando outras espécies de Rhamphastos e R. dicolorus sendo parasitado por Menacanthus balfori Waterson, 1915 e Myrsidea victrix Waterson, 1915. Price \& Weckstein (2005) relataram a presença de $A$. cancelosus parasitando Ramphastos dicolorus em Nova Teutônia, Santa Catarina, Brasil.

\section{Degeeriella sp.}

Material examinado: 2 machos e 5 fêmeas, em Falco sparverius Linnaeus, 1758 (Falconiformes, Falconidae), 03.I.2000, Teixeira, R.H.F., col. PZMQB.

Nota. Price et al. (2003) citaram Degeeriella em sua lista mundial, parasitando esta mesma ave e Colpocephalum subzerafae Tendeiro, 1988, Laemobothrion (Laemobothrion) tinnunculi (Linnaeus, 1758) e Nosopom lucidum (Rudow, 1869) parasitando Falco sparverius.

\section{Goniocotes parviceps (Piaget, 1880)}

Material examinado: 1 macho, em Pavo cristatus Linnaeus, 1758 (Galliformes, Phasianidae), 09.XI.2001, Teixeira, R.H.F., col. PZMQB.

Nota. Emerson \& Elbel (1957) reportaram sobre esta relação parasito-hospedeiro. Goniocotes parviceps também já foi relatada parasitando $P$. cristatus e Pavo muticus Linnaeus, 1766 por Silva et al. (2004) no Brasil. Price et al. (2003) relataram o encontro deste malófago parasitando além de P. cristatus, outras espécies de aves como P. muticus e Lophura nythemera (Linnaeus).

\section{Goniodes pavonis (Linnaeus, 1758)}

Material examinado: 1 macho e 1 fêmea, em Rhea americana Linnaeus, 1758 (Rheiformes, Rheidae) 25.V.2001, Teixeira, R.H.F., col. PZMQB. 
Nota. Goniodes pavonis é uma espécie comumente encontrada em Galliformes segundo Price et al. (2003). Este é o primeiro registro G. pavonis em Rhea americana no sudeste do Brasil.

\section{Heptapsogaster sp.}

Material examinado: 2 fêmeas, em Cariama cristata Linnaeus, 1766 (Gruiformes, Cariamidae) 09.XI.2001, Teixeira, R.H.F., col. PZMQB.

Nota. Brum et al. (2003) identificaram Heptapsogaster sp. como Chelopistes sp. Price et al. (2003) relataram C. cristata sendo parasitada por $C$. cristatae, Heptapsogaster frielingi (Eichler, 1941), Tinamotaecola wardi Hellenthal, Price \& Timm, 2002 and Tinamotaecola zyskowskii Price \& Timm, 2002.

\section{Strigiphilus crucigerus Carriker, 1966}

Material examinado: 1 macho, em Othus choliba Vieillot, 1817 (Strigiformes, Strigidae) VI.XI.2001 Teixeira, R.H.F., col. PZMQB.

Nota. Este resultado corrobora com os achados da chave de identificação de Clayton (1990), que reportou que $O$. choliba é o hospedeiro específico de Strigiphilus. Price et al. (2003) relataram o parasitismo por S. crucigerus em Othus choliba crucigerus (Vieillot, 1817), Otus colombianus Traylor, 1952, Otus cooperi (Ridgway, 1878), Otus guatemale (Sharpe,1875), Otus ingens (Salvin, 1897) e Otus watsonii (Cassin, 1848).

Agradecimentos. As instituições Fundação Jardim Zoológico RioZoo, Rio de Janeiro, Brasil e Parque Zoológico Municipal Quinzinho de Barros, Sorocaba, Brasil por permitirem as coletas.

\section{REFERÊNCIAS}

Brum, J. G. W.; A. L. Valente; R. M. M. Paulsen \& G. Muller. 2003. Malófagos parasitos de animais silvestres do Estado do Rio Grande do Sul. Arquivos do Instituto Biológico 70: 197-198.
Clayton, D. H. 1990. Host specificity of Strigiphilus owl lice (Ischnocera: Philopteridae), with the description of new species and host associations. Journal of Medical Entomology 27: 257265.

Dickinson, E. C. 2003. The Howard and Moore Complete Checklist of the Birds of the World. $3^{\mathrm{a}} \mathrm{rd}$ ed. New Jersey, Princeton University Press, 1039 p.

Emerson, S. K. C. \& R. E. Elbel. 1957. New species and records of mallophaga from Gallinaceous bird of Thailland. Proceedings of the Entomological Society of Washington 59: 232-243.

Freitas, M. F. L.; M. C. N. Botelho; A. S. Leite; V. S. Magalhães; A. E. Sobrinho; R. A. Oliveira; M. H. C. C. Oliveira \& J. B. L. Oliveira. 2002. Ectoparasitos de aves silvestres mantidas em cativeiro no estado de Pernambuco, Brasil. Entomologia y Vectores 9: 2533.

Johnson, K. P. \& D. H. Clayon. 2003. The biology, ecology, and evolution of chewing lice. p. 449-476. In: R. D. Price; R. A. Hellenthal; R. L. Palma; K. P. Johnson \& D. H. Clayton. (eds.) The chewing lice: world checklist and biological overview. Illinois Natural History Survey Special Publication 24. X + 501 p.

Price, R. D. \& J. R. Beer. 1963. The species of Colpocephalum (Mallophaga: Menoponidae) known to occur on the Strigiformes. Journal of the Kansas Entomological Society 36: 58-64.

Price, R. D. \& J. R. Beer. 1965. A Review of Ciconiphilus Bedford (Mallophaga: Menoponidae). Canadian Entomologist 97: 657666.

Price, M. A. \& O. H. Graham. 1997. Chewing and sucking lice as parasites of mammals and birds. Technical Bulletin No. 1849, USDA, Agricultural Research Service. 257 p.

Price, R. D.; R. A. Hellenthal; R. L. Palma; K. P. Johnson \& D. H. Clayton. 2003. The Chewing Lice: World checklist and Biological overview Ilinois Natural History Survey Special Publication 24. $\mathrm{x}+501 \mathrm{p}$.

Price, R. D. \& J. D. Weckstein. 2005. The genus Austrophilopterus Ewing (Phthiraptera: Philopteridae) from toucans, toucanets, and araçaris (Piciformes: Ramphastidae). Zootaxa 918: 1-18.

Sick, H. 1997. Ornitologia Brasileira. $2^{\mathrm{a}}$ ed. Rio de Janeiro, Nova Fronteira, $862 \mathrm{p}$.

Silva, S. O.; H. H. Oliveira; R. H. F. Teixeira \& N. M. Serra-Freire. 2004. Malófagos Parasitas de aves campestres cativos do Zoológico Municipal Quinzinho de Barros, Sorocaba, estado de São Paulo, Brasil. Entomologia y Vectores 11: 333-339.

Valim, M. P.; R. H. F. Teixeira; M. Amorim; N. M. Serra-Freire. 2005. Malófagos (Phthiraptera) recolhidos de aves silvestres no Zoológico de São Paulo, SP, Brasil. Revista Brasileira de Entomologia 49: $584-587$. 